\title{
Computation of Atomic Energy Levels: Spectrum of Singly- Ionized Tantalum (Ta II)
}

\author{
Richard E. Trees, W. F. Cahill, and P. Rabinowitz ${ }^{1}$
}

\begin{abstract}
Energies and wave functions of atomic systems can be calculated as the characteristic values and vectors of matrices in accordance with long-established procedures. Coding for carrying out these computations on the Standards Eastern Automatic Computer is described. Some preliminary values are given for the energy levels of the spectrum of singly-ionized tantalum ( $\mathrm{Ta}$ II).
\end{abstract}

\section{Introduction}

Calculations of atomic energy levels have been helpful in expediting the analysis of experimental data. $^{2}{ }^{3}$ However, except in the simplest cases, the numerical work is excessive for hand computation, and the calculations have had very limited applicability. It is possible to overcome this limitation by utilizing digital computers. A calculation of the levels of the low even configurations in the spectrum of singly-ionized tantalum (Ta II) is given. This is a typical example of a calculation that is too difficult for hand computation, although fairly simple as compared with others in the field of complex spectra.

\section{Statement of Problem}

Spectra in which configuration interaction and spin-orbit coupling are both important are considered; the basic theory for these spectra is well established. ${ }^{45}$ According to this theory, energies of levels with the same total angular momentum (i. e., the same "J-value") are the eigenvalues of a matrix, which is here designated by $\left(a_{i j}\right)$. The $J$-value is omitted for brevity, but there will be one of these

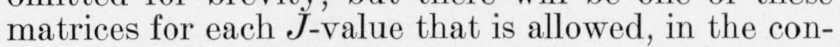
figurations of the spectrum under consideration. The configurations occurring in Ta II are $d^{4}, d^{3} s$, and $d^{2} s^{2}$; these give rise to matrices of orders $9 \times 9$, $12 \times 12,21 \times 21,15 \times 15,15 \times 15,6 \times 6$, and $3 \times 3$ for the allowed $J$-values $0,1,2,3,4,5$, and 6 , respectively.

Each matrix element is a linear combination of a limited number of "parameters", $p^{(k)}$, multiplied by known "coefficients" $c_{i j}^{(k)}$ (the parameters are the same for all $J$-values).

$$
a_{i j}=\sum_{k} c_{i j}^{(k)} p^{(k)} .
$$

\footnotetext{
1 Present address: Weizmann Institute of Science, Rehovot, Israel

2 R. E. Trees and M. M. Harvey, J. Research NBS 49, 397 (1952) RP2378

3 M. Gehatiah, Phys. Rev. 94, 618 (1954)

W. F. Meggers has recently located the $c^{2} \mathrm{D}(3 / 2)$ level of $\mathrm{Hf}$ II at 30595 , less than $100 \mathrm{~K}$ away from Gehatiah's calculated position of 30692 .

${ }_{4}$ E. U. Condon and G. H.Shortley, The theory of atomic spectra (Cambridge University Press, London, 1951).

${ }_{5}$ G. Racah, Pıys. Rev. 62, 438 (1942); 63, 367 (1943); 76, 1352 (1949). Referred to as R II, R III, and R IV, respectively.
}

These linear forms can be derived in a straightforward manner by use of methods developed by Racah (see footnote 5). For commonly occurring configurations, the calculation of any one element of the "coefficient matrix" $\left(c_{i j}^{(k)}\right)$ involves at most the multiplication of a few factors, which are given in readily available tables. ${ }^{6}$ The parameter associated with a given coefficient matrix is defined in theory as a radial integral (Slater integral, spin-orbit integral, etc.) (see footnote 4). This can be evaluated if radial wave functions are determined by the method of self-consistent fields, but the work involved in this procedure is great. In the present calculations, the parameters are adjusted by least-squares to get best agreement with levels that have already been identified experimentally. This limits the use of the theory to spectra in which the analysis of the experimental data is already well started. This limitation may also be removed when computers are used to carry out self-consistent field calculations for complex spectra such as Ta II.

The coefficient matrices for the electrostatic interaction in the configurations of Ta II are given in $\mathrm{R}$ II and $\mathrm{R}$ III. For each $J$-value there are 16 of these associated with parameters identified by the letters $A, B, C, G_{2}, H_{2}$, and $\alpha$ (see table 2$){ }^{7}$ In addition to these, there are three parameters $\zeta$ associated with the coefficient matrices of the spin-orbit interaction. The latter coefficient matrices have been given for the configurations $d^{2} s^{2}$ (see footnote 4) and for $d^{4}$ also. ${ }^{8}$ The matrices for $d^{3} s$ have been computed by Trees; for the sake of brevity, the results are not given here.

Recapitulating, there are 19 different parameters, and for each of the seven $J$-values there are $19 \mathrm{co}-$ efficient matrices, one associated with each parameter. ${ }^{9}$ Though the orders of the coefficient matrices

\footnotetext{
6 Because there are often many elements, it will nevertheless require much time and effort to set up these coefficient matrices correctly; probably automatic com-

7 For a discussion of the parameter $\alpha$ and other advances in atomic theory not
puters can be utilized in this phase of the work. 7 For a discussion of the parameter $\alpha$ and other advances in atomic theory not
covered in footnotes 4 and 5, see G. Racah, Kungl. Fysiografiska Sallskapets Handlingar N. F. 65, 31 (1955). (Proceedings of the Rydberg Centennial Conference on A tomic Spectroscopy).

H. Greyber, Ph. D. thesis, University of Pennsylvania, 1953 (unpublished) ${ }^{9}$ It is convenient to use the code of section 6 to calculate the $g$-values; to do this the $L S$-coupling $g$-values are included as an extra coefficient matrix, the "parameter" associated with the $g$-value always being zero. Therefore, 20 parameters were actually used for Ta II
} 
are the same as those already given for the appropriate $J$-value, there are very few nonzero elements in most of the coefficient matrices; the only coefficient matrices with large numbers of nonzero elements are those associated with the two parameters $\zeta\left(d^{4}\right)$ and $\zeta\left(d^{3} s\right)$.

\section{Eigenvalues and Eigenvectors of Real Symmetric Matrices}

Preliminary estimates of the parameters $p^{(k)}$ are made by using methods given in section 7.1., and the matrix elements are then calculated according to formula (1). This can be done by hand computation; or the coding described in section 5 can be used to generate the matrices on the National Bureau of Standards Eastern Automatic Computer (SEAC). The eigenvalues and eigenvectors of the matrices are then to be determined.

A SEAC code that will do this is based on the Duncan-Collar iteration procedure. ${ }^{10}$ The use of this code with matrices up to order $12 \times 12$ has been briefly reported. ${ }^{11}$ The code can handle matrices with orders up to $25 \times 25$. The major disadvantage of this code is that it requires too much machine time for regular use when all eigenvalues of several high-order matrices are desired. ${ }^{12}$ Because of this, no effort was made to determine all the eigenvectors and eigenvalues for the matrices of Ta II set up with the preliminary values of the parameters. No matrices were set up with the final values obtained for the parameters. ${ }^{13}$

\section{Least-Squares Adjustment of Parameters}

The matrices set up with preliminary values of the parameters $p^{(k)}$ have the eigenvalues $\lambda^{(m)}$ and the eigenvectors $X^{(m)}$ determined (the components of a vector will be indicated by $x_{i}^{(m)}$ ) as described in section 3. The eigenvalues are approximated as a function of the parameters by the following linear formula, which will be accurate to first order, in the difference between the parameters and their preliminary values:

where

$$
\lambda^{(m)}=\sum_{k} d_{(k)}^{(m)} p^{(k)},
$$

$$
d_{(k)}^{(m)}=\sum_{i j} x_{i}^{(m)} c_{i j}^{(k)} x_{j}^{(m)}
$$

10 The matrix is modified to obtain successive values by the method given by W. M. Kincaid, Quart. Appl. Math. 5, 320 (1947).

i1 D. E. Mann, L. Fano, W. F. Cahill, and T. Shimanouchi, J. Chem. Phys. 22, 764 (1954).

12Appreciation is expressed to W. G. Hall for running SEAC to get the energy values in Ta II reported in section 7.2. He also obtained values in the first spectrum of tungsten (W I) for G. Racah; a report on the latter work, which involved matrices with orders up to $25 \times 25$, has been given in footnote 7 . On the basis of his work, Hall has estimated that the average time required to iterate for on eigenvalue and eigenvector is about $5 \mathrm{~min}$ for a $10 \times 10 \mathrm{matrix}, 20 \mathrm{~min}$ for a $20 \times 20$ matrix, and $50 \mathrm{~min}$ for a $25 \times 25$ matrix.

$13 \mathrm{~K}$. Goldberg has a code that determines only the eigenvalues; this code is similar to that described by W. Givens, ORNL 1574 (1954). It can determine the eigenvalues of matrices up to order $30 \times 30$; the time required for a $30 \times 30$ matrix is $25 \mathrm{~min}$. About $10 \mathrm{~min}$ are needed for a $20 \times 20$ matrix. This code is being extended to give the vectors; it is expected that the extended code will be able to handle matrices of orders up to $23 \times 23$.
The coefficients $c_{i j}^{(k)}$ are the ones already used in (1). The linear formulas (2) can be set up by hand; or the coding described in section 6 can be used to generate them on SEAC. A least-squares adjustment can then be made to find new values of the parameters $p^{(k)}$, which will make the linear forms (2) agree best with a corresponding set of observed experimental values.

A general-purpose orthonormalizing code has been set up for SEAC, ${ }^{14}$ and it can be used to carry out least-squares calculations. The coefficients $d_{\left(\begin{array}{c}(m) \\ k\end{array}\right) \text {, for }}$ fixed " $k$ ", are regarded as components of a vector in a space with the same dimensionality as the number of observations to be fitted. For successive $k$-values, each vector is orthogonalized to all preceding vectors with smaller $k$-values. The observed values, regarded as an additional vector, are then expanded in terms of this set, and the residues that remain after the expansion will be the least-squares deviations. The process can be interrupted before the orthonormalization is completed for all $k$-values, so that the least-squares calculation can be carried out, say, first by omitting and then by including the last parameter, without making two separate calculations. As many sets of observations may be fitted as desired in a single calculation. Consequently, different possibilities for correlating observations with theory may be tested. An observation may be omitted from the calculation by inserting a new "weight vector" in which the component corresponding to the deleted observation is given the weight zero. The vectors must be "augmented" in these applications (see footnote 14), so that the leastsquares values of the parameters will be recorded by SEAC (along with the deviations between theory and experiment). This augmentation reduces the capacity of the code when used with the high-speed memory; with 18 adjustable parameters, a maximum of 22 observations could be fitted, whereas with 9 adjustable parameters, 63 observations could be fitted. However, an alternative slightly slower code is available if external tapes are used, and in this the only limitation is that the sum of the number of observations and the number of parameters be less than 256.

The Ta II problem was worked in an approximation where first 8 and then 9 parameters were adjusted (i. e., results were obtained with and without the final parameter $\alpha$ included). There were $39 \mathrm{ob}-$ served values, but the number of these was reduced in successive calculations. As a check, both the observed values and the original deviations from these values were fitted. It required about $20 \mathrm{~min}$ of machine time for each of these calculations as compared to about $9 \mathrm{hr}$ required in an earlier hand computation, where the normal equations were first obtained and then solved by elimination. The sults are described in section 7.3.

14 P. Davis and P. Rabinowitz, J. Assoc. Comp. Mach. 1, 183 (1954). 


\section{Data Preparation-Matrix Inputs}

A code has been set up that will generate all matrices with a single input of the parameter values. It uses the internal memory and can generate matrices of order up to $38 \times 38$. Checks in the code insure that the matrix generated is correct on wire and ready as an input for the coding described in section 3. For the Ta II work, all matrices could be generated in 30 to 60 min of machine time.

When a problem is first started, it is necessary to type many coefficient matrices (20 for the Ta II work) for each final matrix generated, and it would seem simpler to evaluate the elements (1) with a desk computer and type the final matrix directly. ${ }^{15}$ However, only the nonzero coefficients need be typed, and as there are usually very few of these, the total work involved is about the same. ${ }^{16}$ Once the coefficient matrices are typed, final matrices can be generated for as many sets of parameter values as desired. In any one problem, matrices must be set up with at least two sets of parameter values, so an appreciable amount of time will be saved. ${ }^{17}$ The same coefficient matrices are used to generate the linear formulas (2) with the coding described in section 6 , and in relation to this work, the time required to type the coefficient matrices is a negligible factor.

The coefficient matrices used with this code must have no more than 56 nonzero elements, but larger coefficient matrices can be inserted piecemeal by associating extra parameters (which will be given the same value) with each part. To allow for this possibility, the code has been written for 24 parameters, which is probably a larger number than would ever be needed. The largest coefficient matrix encountered in the Ta II problem had only 36 nonzero elements.

\section{Data Preparation - Least-Squares Inputs}

A code has been set up that will generate the coefficients, formula (3), that enter into the linear formula for the eigenvalue, formula (2). Instructions that determine the number of independent parameters desired in the linear formula outputs are first fed in; frequently, as here, parameters having the same symbol are regarded as identical in all configurations in which they occur, but other approximations are also used. All vectors for which linear formulas are desired are then fed into the internal memory; there is space for 582 vector com-

15 The time required to set up the code was naturally far in excess of the time needed to do the work with hand computers. The expenditure of this time is justified only because it is expected that the same coding will be used subsequently in many similar problems.

16 The last four digits of each coefficient are used to specify the position in the matrix; this leaves seven digits for the coefficient itself. In the present work only four only mean deviation between theory and experiment more than a hundred times as mean

great.

The same set of coefficient matrices can often be used to generate final matrices for other spectra that have the same, or related, configurations. The coefficient matrices used in the Ta II work also apply to the configurations $d^{8}, d^{7} s$, and $d^{6} s^{2}$, and they have been used to generate the matrices for the first spectrum of ruthenium (Ru I) as a start toward assisting with the analysis of this spectrum. ponents (all vectors of a $24 \times 24$ matrix). ${ }^{18}$ 'The coefficient matrices are then fed in, and for each one fed in (or for each group of consecutive matrices, depending on the instructions), as many coefficients of linear formulas print out as there are vectors in the vector input (the maximum number of possible outputs is 24). For the Ta II work, the 39 linear formulas in nine parameters were computed in less than 60 min of machine time; originally this work was carried out with a desk computer, and the time required was of the order of $60 \mathrm{hr}$.

The coding just described groups coefficients of the same parameter only if they are in the same matrix. For use in the least-squares code of section 4 , coefficients of the same parameter must be grouped together without regard to the $J$-value of the matrix in which they occur. The second part of this code sorts out the preceding coefficient outputs. A remaining uncorrected deficiency is that it does not give an output ready to be used as an input for the orthonormalization code of section 4 ; the augmenting and check sums were inadvertently overlooked, so the results had to be outscribed, amplified, and reinscribed to get the correct input. This sorting can be carried out only for problems where the number of coefficients, formula (3), is less than 672 . With 15 parameters (which may be the least number that can give good agreement in Ta II) only 44 observations can be handled (and 39 of 81 possible levels have already been observed in Ta II). This limitation is a very difficult one to overcome without altering the construction of the computer itself. However, it is always possible to combine two partial sortings and set up by hand the final input for the least-squares code.

It would be convenient at this point to carry out a few manipulations with the sorted linear formulas, but the potentialities for doing this have not yet been fully explored. However, supplemental coding has been made that will evaluate the linear formulas, and also the differences between the formulas and a set of observed values, for as many sets of parameters as desired.

\section{Results for Ta II}

\subsection{Preliminary Estimates of Parameters}

The spectrum of Ta II is very complicated because of the presence of the three overlapping configurations of $5 d^{4}, 5 d^{3} 6 s$, and $5 d^{2} 6 s^{2}$. The analysis of the experimental data ${ }^{19}$ is far from complete, as only 39 of 81 possible levels in these configurations have been identified. $J$-values are assigned to all identified levels, but the configuration and $L S$-coupling desig-

18 With some amplification, the coding could be used to obtain nondiagonal elements of the transformed coefficient matrices (as well as the diagonal elements, formula (3)); it is estimated that matrices of order up to $18 \times 18$ might be trans formed with this amplified code. Such a code would be useful in solving the problem referred to in footnote 6 because it is sometimes easiest to set up coeffiproblem referred to in footnote 6 because it is sometimes easiest to set up coefficient matrices in a scheme that does not correspond to observed behavior, and then to use a simple tran

19 C. C. Kiess, G. R. Harrison, and W. J. Hitcheock, J. Research NBS 44. 245 (1950) RP 2075. 
nations have been made for only 26 of them. However, the configuration assignments of six of the lowest levels have been uncertain. Kiess (see footnote 19) had assigned these levels to the ${ }^{3} \mathrm{~F}$ and ${ }^{3} \mathrm{P}$ terms of the $5 d^{2} 6 s^{2}$ configuration, but this assignment put the ${ }^{3} \mathrm{P}$ term below the ${ }^{3} \mathrm{~F}$ term, whereas it would be expected to be much higher on the basis of a simple $L S$-coupling theory. It would have been reasonable to assign the ${ }^{3} \mathrm{~F}$ term to the $5 d^{3} 6 s$ configuration, but this would leave the ${ }^{3} \mathrm{~F}$ term in $5 d^{2} 6 s^{2}$ still unidentified and force one to conclude that the lowest levels of this spectrum, corresponding to the $5 d^{2} 6 s^{2}{ }^{3} \mathrm{~F}$ term, were completely unknown. ${ }^{20}$ In any case, it was certain that configuration and $L S$ coupling assignments would be approximations at best, so that it would be very hard to decide what experimental values should be correlated with theory in estimating preliminary values for the parameters.

Because of this, the theory was first applied to the experimental values ${ }^{21}$ in the simpler, almost completely analyzed spectrum of singly-ionized hafnium (Hf II), to get some idea of the values that would be expected for the parameters in spectra with $5 d$ electrons; no calculations had been published for these spectra at that time. The Hf II parameters were not evaluated by least-squares, but the values obtained agreed well with those recently published by Gehatiah (see footnote 3 ). ${ }^{22}$

Preliminary parameter values were then estimated for Ta II by adjusting the Hf II values to get rough agreement with a few of the best identified levels of the Ta II spectrum. The magnitude of the readjustment was kept reasonably consistent with what would be expected from the known behavior of the parameters in spectra with $3 d$ - and $4 d$-electrons. However, some of the parameters were poorly adjusted, and acceptable preliminary values of the

${ }^{20}$ In commenting on this, Kiess noted that too few strong transitions were left unidentified in his line list to give much weight to this possibility (private communication). The assignment made by Kiess was originally made also by P. F. A. Klinkenberg, G. J. van den Berg, and J. C. van den Bosch, Physica 16. 861 (1950), but they later favored the second possibility that the lowest levels had 861 (1950), but they later favored the second possibility that the lowest levels had not yet been identified (Physica 17, 167 (1951)); theory was used to demonstrate versity of Amsterdam, 1951 (unpublished)). His calculation is much too simpliversity of Amsterdam, 1951 (unpublished)). His calculation is much too simpli-
fied to decide the point, however, and the calculation given here shows conclufied to decide the point, however, and the calcu
sively that the first alternative is the correct one.

sively that the first alternative is the correct one. 22 Gehatiah's parameter values for Hf II are:

$$
B=435, C=1,530, G_{2}=2,818, \mathrm{H}_{2}=482 \text {, and } \zeta=1,336 .
$$

Analogy with results for $3 d$ - and $4 d$-electrons suggests that the Ta II parameters should more nearly equal to the parameters of Ta I or W I (excepting the spin-orbit parameter $\zeta$ for the latter spectrum) than to those of Hf II. Some recent unpublished evaluations of parameters in these two first spectra are interesting. Racah has given the final parameter values that he obtained in the $5 d^{4} 6 s^{2}$ and $5 d^{5} 6 s$ configurations of $\mathrm{W}$ I:

$$
B=371, C=1,900, G_{2}=2,700, \mathrm{H}_{2}=434, \zeta=2,089 \text { and } \alpha=46 .
$$

His calculations are briefly described in footnote 7. The Ta I calculations are for the $5 d^{3} 6 s^{2}$ configuration and neglect configuration interaction; they have been carried out with and without the $L(L+1)$ correction (see $\mathbf{R}$. E. Trees, Phys. Rev. 92, 308 (1953) and also T. Kamei, Phys. Rev. 99, 789 (1955):

$$
\begin{gathered}
B=345, \mathrm{C}=1,289, \zeta=1,657, \alpha=112 . \\
B=278, \mathrm{C}=2,100, \zeta=1,650 .
\end{gathered}
$$

The neglect of configuration interaction in the Ta I calculations is probably not fully justified, which may account for the value of $\alpha$ being larger than expected. parameters were still obtained, so it is likely that this readjustment is not critical. Some details of the procedure will be given, however, mainly to bring out significant features of the parameter behavior. The preliminary parameter values are given in calculation I of table 1 , and the final leastsquares-adjusted values of the parameters are given in calculation II.

The preliminary values for parameters $B$ and $C$ were assumed to be 20 percent greater than the values in Hf II, although an increase of only about 15 percent would be expected from analogy with $3 d$ electron spectra. Calculation II shows not only that this overestimated $B$ and $C$, but also what is more important, that the final ratio of $B$ to $C$ in Ta II is about 8 percent smaller than the ratio in Hf II (it is 25 percent greater than the ratio for the W I parameters (see footnote 22). This ratio has a more constant value in spectra with $3 d$ - and $4 d$ electrons (see footnote 22). The ratio may vary in spectra with $5 d$-electrons, but it is also possible that a nearly constant ratio would be obtained in these spectra if a higher-order approximation were used, as suggested at the end of this paper.

The preliminary estimate $G_{2}=2,000$ was made to favor the assumption that the lowest identified ${ }^{3} \mathrm{~F}$ term belonged to the $5 d^{3} 6 s$ configuration, even though this assumption was not considered correct. Calculation II shows that $G_{2}$ should have been given the value found in Hf II. Analogy with $3 d$-electron spectra indicates that $G_{2}$ should be slightly larger in Ta II than in Hf II, but the increase is much less than expected for $B$ and $C$.

The value $\zeta=2,100$ is a considerable overestimate; calculation II indicates that a value slightly larger than the value applicable in Ta I should have been used (see footnote 22), which is what would be expected from analogy with $3 d$-electron spectra.

To estimate the interaction parameter $\mathrm{H}_{2}$, the ${ }^{3} \mathrm{~F}$ and ${ }^{3} \mathrm{P}$ matrices were set up in $L S$-coupling by using all the preceding preliminary parameter evaluations. The lowest terms of these matrices were expected to be very sensitive to the value of the parameter $H_{2}$, so that errors in estimating the other parameters would not be too important. A value of 500 was assumed for $\mathrm{H}_{2}$, as it was expected that the parameter would have a value in Ta II that would nearly equal that in Hf II. Analogy with the behavior shown in the $3 d$-electron spectra would lead one to expect that the value in Ta II might be smaller than in Hf II, but as this integral depends strongly on the overlapping of $d$ - and $s$-wave functions, the analogy would not be very reliable. The eigenvalues of the two $5 \times 5$ matrices were obtained on SEAC with the coding described in section 3 . The lowest ${ }^{3} \mathrm{P}$ term was found to be $800 \mathrm{~K}$ above the ${ }^{3} \mathrm{~F}$ term, instead of $1800 \mathrm{~K}$ below as observed. It was decided that an extra 10percent increase in the value of $\mathrm{H}_{2}$ would be justified, and that this, together with the inclusion of secondorder spin-orbit effects, would explain the observations with reasonable parameter values. 
TABLE 1. Configurations $d^{2} s^{2}, d^{3} s$, and $d^{4}$ of Ta II in intermediate coupling

Levels are identified by their $J$-values and by the label number, which is the order in energy sequence.

Calculation I: Solutions of matrices for preliminary values of the parameters.

Calculation II: Solution from linear formulas; parameters evaluated by least-squares, with the levels 18500 and 23295 omitted.

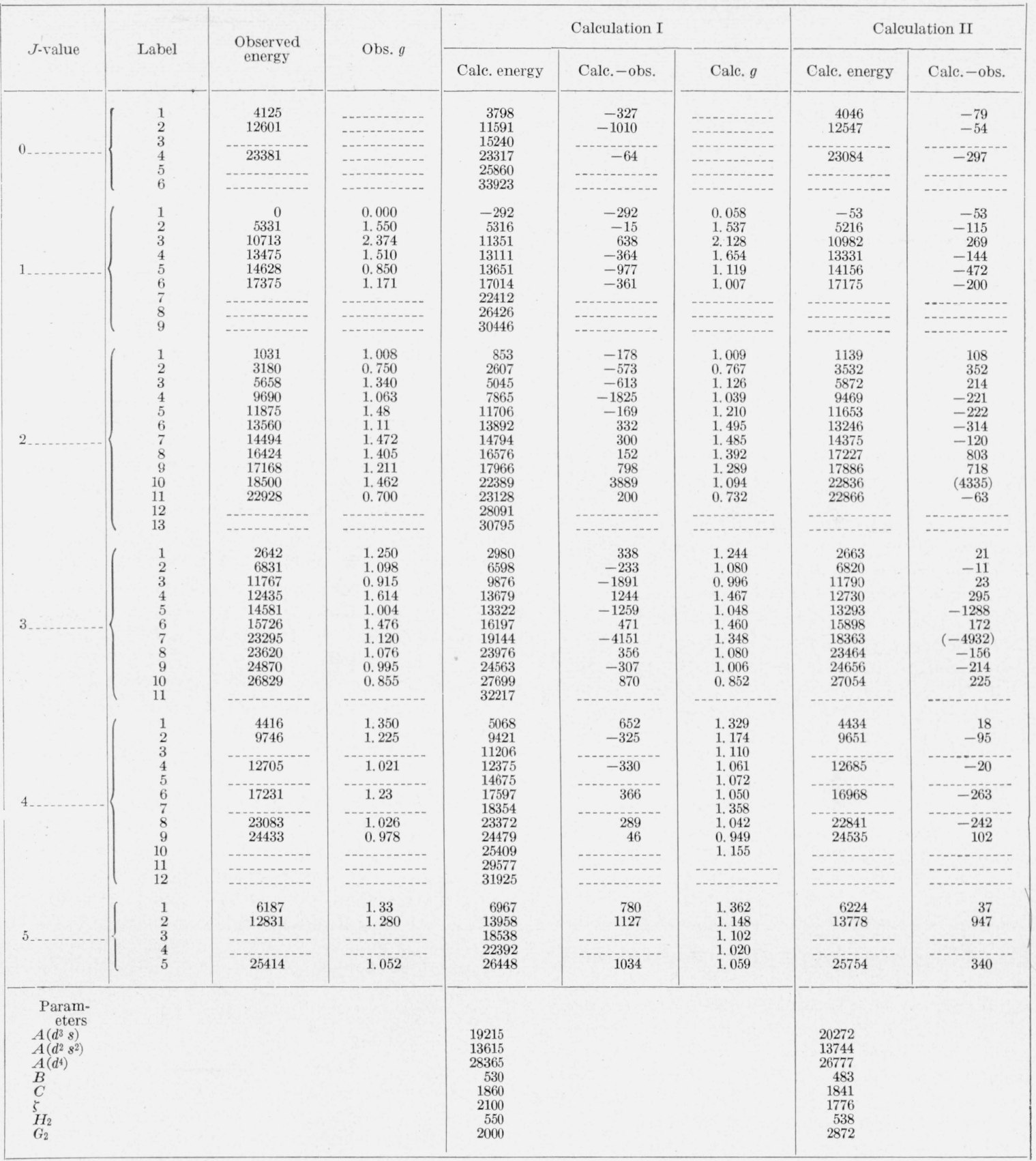

\subsection{Preliminary Calculation of Energy Levels}

By using these preliminary values for the parameters, matrices were set up for the Ta II problem, as outlined in section 2. (The coding in section 5 was not then available.) The eigenvalues and eigenvectors of these matrices were then calculated on SEAC, as described in section 3. The results are given in calculation I of table 1. Partly because the eigenvectors obtained in this calculation are based on preliminary values for the parameters, no attempt has been made to give the conventional $L S$-coupling designation for the levels, and the levels have been identified by the $J$-values alone; the assignments given to some of these levels by Kiess (see footnote 19) are, however, roughly confirmed. 
Although the preliminary parameters were estimated without making any effort to assign the majority of the observed levels to any theoretical state, a good correlation can be established between observed energy levels and calculated eigenvalues, and between the corresponding observed and calculated $g$-values. There are, however, three observed levels with $J=2(16424,17168$, and 18500$)$ in a region where there are only two calculated (16576. $17966)$; the $g$-values indicate that the level which cannot be correlated with theory is the one observed at 18500. Similarly, there are three observed levels with $J=3(23295,23620$, and 24870$)$ in a region where two are calculated (23976 and 24563); in this case the $g$-values indicate that the level which cannot be correlated with theory is the one observed at 23295. The levels with $J=3$ observed at 12435 and 14581 were correlated with the respective calculated values 13679 and 13322 . This is the only instance where the calculated and observed values were not correlated in the same sequence with respect to energy. This correlation was made when it was found with better parameter estimates that the linear formulas for the eigenvalues indicated that their energies would cross. This correlation could have been made more easily by noting that it makes the $g$-values agree much better, but this criterion would also call for reversing the correlations of the fifth and sixth levels with $J=2$, and this was not done. Within the limits of accuracy of this preliminary calculation, the only other correlations that seem uncertain are the ones connecting the level observed at 12705 with the level calculated at 12375 , rather than the ones calculated at 11206 or 14675 , and also the correlation of 17231 with 17597 rather than with 18354 . Both these correlations worked well in subsequent least-squares calculations. However, the leastsquares calculations show that agreement would be improved if the level observed at 16424 were disregarded, rather than the one at 18500 . It is likely that other possibilities still remain, that might give better agreement.

\subsection{Least-Squares Estimates of Parameters}

Linear formulas for the eigenvalues were set up by hand (the coding of section 6 was not then available) in an approximation with nine adjustable parameters; the parameter $\alpha$, which was neglected for the preliminary estimates, is the additional parameter. Least-squares calculations were then carried out on SEAC, with and without the parameter $\alpha$, as described in section 4. These calculations will be described briefly; the parameter values that were obtained are given in successive columns of table 2 , labeled to correspond to the descriptions.

(I) The parameters were determined by using all 39 observed levels with the correspondence between theory and observation the same as that already given in calculation I of table 1. The purpose was to verify, what was already fairly certain, that no readjustment of the parameters would give a good fit for all 39 observations. In this calculation the level
TABLE 2. Least-squares evaluations of the parameters of $d^{2} s^{2}, d^{3} s$, and $d^{4}$ of Ta II

The parameters $A$ determine the centers of gravity of the three configurations; $B, C, \zeta$, and $\alpha$ would be specified independently in all three configurations in higher approximations than the one given here. The interaction parameter $G_{2}\left(d^{4}-d^{2} s^{2}\right)$, has been taken equal to the exchange integral $G_{2}\left(d^{3} s\right)$ and designated $G_{2} ;$ the two interaction integrals $H_{2}\left(d^{4}-d^{3} s\right)$ and $H_{2}\left(d^{3} s-d^{2} s^{2}\right)$ are taken equal and designated $\mathrm{H}_{2}$.

The first of a pair of determinations grouped together omits the $L(L+1)$ cor rection, the second includes it. A correspondingly numbered section of the text specifies the observations included in making each pair of calculation.

\begin{tabular}{|c|c|c|c|c|c|c|c|c|}
\hline \multirow{2}{*}{ Parameter } & \multicolumn{8}{|c|}{ Calculation- } \\
\hline & \multicolumn{2}{|c|}{ I } & \multicolumn{2}{|c|}{ II } & \multicolumn{2}{|c|}{ III } & \multicolumn{2}{|c|}{ IV } \\
\hline$A\left(d^{3} s\right)$ & 19505 & 19303 & 20272 & 20168 & 20188 & 20140 & 20446 & 20388 \\
\hline$A\left(d^{2} s^{2}\right)$ & 12843 & 12892 & 13744 & 13785 & 13614 & 13641 & 13636 & 13957 \\
\hline$A\left(d^{\dagger}\right)$ & 26563 & 26095 & 26777 & 26498 & 26468 & 26341 & 26830 & 26356 \\
\hline$B$ & 461 & 424 & 483 & 462 & 474 & 464 & 479 & 447 \\
\hline$C$ & 1915 & 2088 & 1841 & 1945 & 1897 & 1947 & 1824 & 2012 \\
\hline$G_{2}$ & 2496 & 2476 & 2872 & 2863 & 2859 & 2856 & 2924 & 2918 \\
\hline & 1980 & 2008 & 1776 & 1800 & 1786 & 1798 & 1843 & 1874 \\
\hline $\mathrm{H}_{2}$ & 497 & 502 & 538 & 541 & 538 & 539 & 521 & 544 \\
\hline$\alpha \ldots$ & $\ldots$ & -35 & -.. & -19 & -.. & -10 & $\ldots$ & -35 \\
\hline
\end{tabular}

at 18500 had a deviation of 3817 , and the deviation for the level at 23295 was -4294 . The level at 12831 had the next largest deviation of 1316 , which is an order of magnitude smaller. The mean deviation was \pm 1048 ; the mean deviation, exclusive of the two badly fitting levels, was \pm 515 .

The inclusion of the $L(L+1)$ correction produced a negligible improvement in the mean deviations, and the correction was negative $(\alpha=-35)$, whereas all the evidence so far accumulated indicates that it should be positive. With the $L(L+1)$ correction the mean deviation for all levels was reduced to $\pm 1,038$, and the deviation, excluding the two badly fitting levels, was \pm 510 .

(II) The levels at 18500 and 23295 were omitted from the least-squares calculation; the deviations for this calculation are given in calculation II of table 1 . The mean deviation of this calculation is \pm 371 : As this is considerably less than the value \pm 515 for calculation (I), described in the previous paragraph, it indicates that the parameters of the latter calculation were influenced by the inclusion of the two badly fitting levels. Comparison of the parameters themselves also indicates this. In this calculation there are three deviations (for the levels 16424, 14581 , and 12831) that exceed twice the mean deviation of all levels fitted.

Again, when the $L(L+1)$ correction is included, the mean deviation is reduced only slightly (to the value \pm 364$)$ and the correction is negative $(\alpha=-19)$. Most of the improvement produced by including the $L(L+1)$ correction can be attributed to the slightly better agreement obtained for the badly fitting level 12831 , which has its deviation reduced to 858 ; if the mean deviation is computed without this level the value \pm 371 would be replaced by \pm 341 , and the value \pm 364 replaced by \pm 340 . The inclusion of the $L(L+1)$ correction is therefore not justified.

As calculations III and IV indicate, the inclusion of three badly fitting levels has not had much effect on the agreement obtained for the other levels. Also, there seems to be no possibility of including 
the $L(L+1)$ correction and getting an appreciable improvement. The best calculation that can be offered at this time is that in calculation II of table 1. Possibilities for improving this calculation will be given in the discussion.

(III) The levels 18500, 23295, and 12831 were omitted from the least-squares calculation. The mean deviation was \pm 335 , which is only a little less than the value \pm 341 obtained in (II). Inclusion of the $L(L+1)$ correction produced negligible improvement, as the deviation was reduced only to \pm 333 ; the correction was still negative $(\alpha=-10)$. It would have been difficult to justify the omission of the level 12831 if this calculation had given much better agreement.

(IV) Eleven levels were omitted in this calculation. These omissions consist of the two pairs, 18500 and 22928 , and 12435 and 14581 , omitted partly because the calculated eigenvalues were close and it seemed possible, therefore, that the linear formulas might not apply; the two triplet groups 23295,23620 , and 24870 , and 16424,17168 , and 18500 , which were omitted because of uncertainties in the correspondence between theory and experiment; and the levels 23381 and 12831 , omitted because they had rather large deviations that tended to be improved by a negative $L(L+1)$ correction. The mean deviation of this calculation was \pm 170 (the mean deviation of the same terms in calculation (II) is \pm 200 ). The only large deviation was -491 for the level 14628 . With the $L(L+1)$ correction included, the mean deviation was reduced to \pm 151 , due mainly to a decrease in the deviation for the level 14628 to -306 . Again the sign of the correction was negative $(\alpha=-35)$.

\section{Discussion}

Results obtained in spectra with $3 d$ - and $4 d$-electrons indicate that when the $L(L+1)$ correction is used the mean deviation between theory and experiment will generally be less than $200 \mathrm{~K}$, and that the sign of the correction will be positive. ${ }^{23}$ Calculation (II) of table 1 should therefore be improved because

\footnotetext{
${ }^{23}$ Racah (see footnote 7) reports a mean error of 411 without the $L(L+1)$ correction, and an error of 229 with the $L(L+1)$ correction in W I; as indicated in footnote 22 , the sign of the $L(L+1)$ correction is positive. By coincidence, the mean error of calculation II, table 1 is $412 \mathrm{~K}$, nearly identical with the corresponding W I value; however, no appreciable improvement was obtained by the use of the $L(L+1)$.correction In Hf $\Pi$, Gehatiah (see footnote 3 ) obtains a mean error of 257 without the use of this correction.
}

a better significant agreement between theory and experiment can probably be obtained.

It is fortunate that the experimental analysis of the Ta II spectrum will be extended by members of the NBS Spectroscopy Section. The calculation given is already accurate enough to furnish considerable help. This analysis may, in turn, help solve the problem of the two excess levels pointed out previously. In a preliminary survey, the switching of $J$-values has been ruled out, as none of the levels concerned are poorly identiifed. The identification of new levels will help to eliminate some of the arbitrariness involved in correlating the observed and calculated values. With more observations a calculation with extra adjustable parameters (suggested below) will also be better justified.

Eigenvalues and eigenvectors of the matrices set up with least-squares values of the parameters will be obtained on SEAC after the coding now underway is completed (see footnote 13). The $g$-values obtained should be much more accurate than those already calculated, and if this is so, the uncertainties in making correlations may be resolved. Knowledge of the exact eigenvalues will show whether or not any of the large errors of the calculation are due to limitations on the validity of linear formulas for the eigenvalues. The linear formulas obtained from these new solutions should be accurate enough so that eigenvalues can be redetermined from them for any additional small changes that are made in the parameters, without setting up new matrices.

The agreement between theory and experiment may also be improved by using more adjustable parameters; $B, C$, and $\zeta$ can be adjusted independently in all three configurations, thus increasing the number of adjustable parameters from 9 to 15 . Theoretically, this should be done, as the effect of differences in these parameters is likely to have the same order of magnitude as the $L(L+1)$ correction. ${ }^{24}$ The difficulty of carrying out least-squares calculations with this number of parameters is great, even with SEAC. Some coding improvements and more experience in the behavior of the codes with this type of problem are required.

${ }_{24}$ See section 4.2 of the reference in footnote 2 , or section 2 of the reference in footnote 7 .

Washington, September 9, 1955. 